\title{
Does Economic Insecurity Really Impact on Gun Violence at US Schools?
}

Pappa, E. ${ }^{1,2 *}$ Lagerborg, A. ${ }^{1}$, and Ravn, M.O. ${ }^{2,3,4}$

March 2018

${ }^{1}$ European University Institute, Department of Economics, Villa La Fonte, Via delle Fontanelle 18, 50014 San Domenico di Fiesole, Italy. ${ }^{2}$ Centre for Economic Policy Research. ${ }^{3}$ University College London, University College London, Drayton House, Gower Street, London WC1E 6BT, UK. ${ }^{4}$ ESRC Centre for Macroeconomics. *e-mail: evi.pappa@eui.eu 
Much research examines the impact of unemployment on crime. The consensus view is that the crime-unemployment link is weak for most types of crimes and inexistent for others including violent crime and murder. ${ }^{\mathrm{i}, i}$ Furthermore, unemployment correlates negatively with most measures of school violence (Supplementary Table 21). Pah et al. (2017) conclude that higher unemployment causes increased risk of school shootings. We argue that the estimated correlation between unemployment and school shootings does not reflect a causal relationship but derives from omitted variables.

We study Pah et al.'s dataset extended with county level observations on school shootings and unemployment. We initially consider the Poisson model:

$$
\mathbb{E}\left(S_{m} \mid u_{m}, m_{s}\right)=e^{\beta_{0}+\beta_{1} u_{m}+\beta_{2} m_{s}}
$$

$S_{m}=$ number of school shootings, $u_{m}=$ unemployment rate, and $m_{s}=$ dummy for the summer months. The data are monthly for 1990-2013.

Consistently with Pah et al., the estimates of $\beta_{1}$ (including geographical fixed effects when analysing subnational data) are statistically significant at each geographical level (Supplementary Table 1).

However, the significance of $\beta_{1}$ is not robust. Pah et al. argue that the frequency of school shootings varies stepwise over time. Table 1 shows that $\beta_{1}$ becomes statistically insignificant when allowing for regime-specific intercepts to control for such slow-moving trends. The same conclusions hold when estimating (1) separately for each sub-period (Supplementary Table 2), or when including common time fixed effects in the sub-national regressions (Supplementary Table 1). Thus, Pah et al.'s conclusions plausibly derive from spurious correlations and/or omitted variables (see Angrist and Pischke, 2009iv).

Contagious effects of shootings offer one possible explanation of the results above. The idea that particularly violent crimes are contagious is an old one (Tarde, $\left.1890^{\mathrm{v}}\right)$. Recently, Towers et al. (2015) vi find that US mass killings and school shootings are contagious.

Supplementary Figure 5 illustrates the number of school shootings and the average fatalities per incident together with the timing of the three deadliest mass shootings in the sample (Luby's, the Virginia Tech and the Sandy Hook shooting). Clearly, the number of school shootings rises persistently after these episodes.

We therefore include controls for past mass shootings in (1), defined as incidents with minimum four fatalities (excluding the perpetrator) carried out by a lone shooter in a public sphere. Table 1 shows that massacres are highly significant in explaining school shootings in a 2-3 year window after their occurrence. Moreover, controlling for contagion, unemployment is insignificantly related to school shootings. Results generalize to the other economic indicators considered by the authors (Supplementary Tables 9-12) and are robust to various definitions of mass shootings (Supplementary Table 6).

The persistent contagion effects are consistent with evidence that many school shooters were inspired by the Columbine and Virginia Tech massacres even several years thereafter (Mother Jones, 10/5/2015 vii). Another possible mechanism generating persistence is increased gun 
sales following massacres (Studdert et al., 2017viii ; Supplementary Table 7) combined with the impact of gun ownership on firearm homicide rates (Siegel, Ross and King, 2013 ${ }^{\mathrm{ix}}$ )

Few things may matter as much to US parents as their off-spring's health and safety, hence, the results of Pah et al. and their interpretation are very important. We argue that the correlation between unemployment and school shootings cannot be given causal interpretation. Mass shootings are better predictors of future school shootings but research has to uncover why such shootings occur in the first place.

i Corman, H. \& Corman, N. J. Law and Econ. 48, 235-66 (2005)

ii Levitt, S.D. J. Econ. Perspect. 18, 163-90 (2004)

iii Pah, A.R. et al. Nat. Hum. Behav. 1, 1-6 (2017)

iv Angrist, J.D. \& Pischke, J.S, Mostly Harmless Econometrics. Princeton University Press (2009)

${ }^{\vee}$ Tarde, G. Penal Philosophy, Boston: Little, Brown (1890)

vi Towers, S., et al. PLoS ONE 10 (2015).

vii Mother Jones, How Columbine Spawned Dozens of Copycats.

https://www.motherjones.com/politics/2015/10/columbine-effect-mass-shootings-copycat-data/

viii Studdert, D.M. et al. Ann. Intern. Med. 166, 698-706 (2017)

ix Siegel, M., Ross, C.R., King, C. Am. J. Public Health. 103, 2098-2105 (2013)

× Duwe, G., Mass Murder in the United States: A History. McFarland \& Co. (2007) 


\section{Materials}

Data. We have used the data of Pah et al (2017) available at https://amaral.northwestern.edu/school_gun_violence/. Seasonally-adjusted unemployment rates were obtained from the Bureau of Labor Statistics (BLS). At the county-level, unemployment rates were only available on a non-seasonally adjusted basis from BLS's Local Area Unemployment Statistics (www.bls.gov/lau), and we seasonally adjusted them using the Census Bureau X13 procedure. Data on mass shootings were obtained from Mother Jones and from Grant Duwe. Data and codes to replicate the analysis in the correspondence are available at: https://sites.google.com/site/alagerborg/research

\section{Author contributions}

All authors contributed equally to the development of the response.

Table 1: Poisson Regression Controlling for Sub-Periods and Mass Shootings

\begin{tabular}{|c|c|c|c|c|c|c|c|c|}
\hline $\begin{array}{l}\text { Geographical Unit } \\
\text { Dependent variable } \\
\text { Mass definition }\end{array}$ & $\begin{array}{c}(1) \\
\text { National } \\
\text { No. } \\
\text { Shootings }\end{array}$ & $\begin{array}{c}(2) \\
\text { Regional } \\
\text { No. } \\
\text { Shootings }\end{array}$ & $\begin{array}{c}\text { (3) } \\
\text { County } \\
\text { No. } \\
\text { Shootings }\end{array}$ & $\begin{array}{c}\text { (4) } \\
\text { National } \\
\text { No. } \\
\text { Shootings } \\
\text { MJ (Top 3) }\end{array}$ & $\begin{array}{c}(5) \\
\text { Regional } \\
\text { No. } \\
\text { Shootings } \\
\text { MJ (Top 3) }\end{array}$ & $\begin{array}{c}(6) \\
\text { County } \\
\text { No. } \\
\text { Shootings } \\
\text { MJ (Top 3) }\end{array}$ & $\begin{array}{c}\text { (7) } \\
\text { National } \\
\text { No. } \\
\text { Shootings } \\
\text { MJ (All) }\end{array}$ & $\begin{array}{c}(8) \\
\text { National } \\
\text { No. } \\
\text { Shootings } \\
\text { Duwe (All) }\end{array}$ \\
\hline Unemployment & $\begin{array}{l}-0.017 \\
(0.043)\end{array}$ & $\begin{array}{l}-0.008 \\
(0.041)\end{array}$ & $\begin{array}{c}0.009 \\
(0.030)\end{array}$ & $\begin{array}{c}0.027 \\
(0.038)\end{array}$ & $\begin{array}{l}0.026 \\
(0.036)\end{array}$ & $\begin{array}{l}0.028 \\
(0.028)\end{array}$ & $\begin{array}{l}0.075 \\
(0.050)\end{array}$ & $\begin{array}{l}0.024 \\
(0.046)\end{array}$ \\
\hline 1992-1994 & $\begin{array}{c}1.939 * * * \\
(0.306)\end{array}$ & $\begin{array}{c}1.936 * * * \\
(0.306)\end{array}$ & $\begin{array}{c}1.931 * * * \\
(0.306)\end{array}$ & & & & & \\
\hline $1994-2007$ & $\begin{array}{c}0.918^{* * *} \\
(0.296)\end{array}$ & $\begin{array}{c}0.925 * * * \\
(0.295)\end{array}$ & $\begin{array}{c}0.956 * * * \\
(0.292)\end{array}$ & & & & & \\
\hline 2007-2013 & $\begin{array}{c}1.541 * * * \\
(0.293)\end{array}$ & $\begin{array}{c}1.525 * * * \\
(0.293)\end{array}$ & $\begin{array}{c}1.510 * * * \\
(0.292)\end{array}$ & & & & & \\
\hline $\begin{array}{l}\text { Within } 1-12 \text { months } \\
\text { of mass shootings }\end{array}$ & & & & $\begin{array}{c}0.615 * * * \\
(0.166)\end{array}$ & $\begin{array}{c}0.629 * * * \\
(0.166)\end{array}$ & $\begin{array}{c}0.614 * * * \\
(0.165)\end{array}$ & $\begin{array}{r}0.007^{* *} \\
(0.003)\end{array}$ & $\begin{array}{c}0.013^{* * *} \\
(0.004)\end{array}$ \\
\hline $\begin{array}{l}\text { Within } 13-24 \text { months } \\
\text { of mass shootings }\end{array}$ & & & & $\begin{array}{c}0.774 * * * \\
(0.171)\end{array}$ & $\begin{array}{c}0.788 * * * \\
(0.170)\end{array}$ & $\begin{array}{c}0.771^{* * *} \\
(0.166)\end{array}$ & $\begin{array}{c}0.012 * * * \\
(0.004)\end{array}$ & $\begin{array}{c}0.012^{* *} \\
(0.005)\end{array}$ \\
\hline $\begin{array}{l}\text { Within } 25-36 \text { months } \\
\text { of mass shootings }\end{array}$ & & & & $\begin{array}{c}0.698 * * * \\
(0.186)\end{array}$ & $\begin{array}{c}0.715^{* * *} \\
(0.182)\end{array}$ & $\begin{array}{c}0.694^{* * *} \\
(0.173)\end{array}$ & $\begin{array}{c}0.003 \\
(0.005)\end{array}$ & $\begin{array}{l}0.006 \\
(0.006)\end{array}$ \\
\hline $\begin{array}{l}\text { Within } 37-48 \text { months } \\
\text { of mass shootings }\end{array}$ & & & & $\begin{array}{c}0.262 \\
(0.202)\end{array}$ & $\begin{array}{c}0.278 \\
(0.200)\end{array}$ & $\begin{array}{c}0.258 \\
(0.196)\end{array}$ & $\begin{array}{l}-0.005 \\
(0.005)\end{array}$ & $\begin{array}{c}0.004 \\
(0.005)\end{array}$ \\
\hline Summer & $\begin{array}{c}-1.107^{* * *} \\
(0.175)\end{array}$ & $\begin{array}{c}-1.101^{* * *} \\
(0.176)\end{array}$ & $\begin{array}{c}-1.108^{* * *} \\
(0.175)\end{array}$ & $\begin{array}{c}-1.131 * * * \\
(0.178)\end{array}$ & $\begin{array}{c}-1.125^{* * *} \\
(0.178)\end{array}$ & $\begin{array}{c}-1.131 * * * \\
(0.178)\end{array}$ & $\begin{array}{c}-1.127 * * * \\
(0.178)\end{array}$ & $\begin{array}{c}-1.070^{* * *} \\
(0.184)\end{array}$ \\
\hline Constant & $\begin{array}{l}-0.620 \\
(0.396)\end{array}$ & $\begin{array}{c}-2.589 * * * \\
(0.395)\end{array}$ & $\begin{array}{c}-6.783^{* * *} \\
(1.103)\end{array}$ & $\begin{array}{c}0.098 \\
(0.220)\end{array}$ & $\begin{array}{c}-1.841^{* * *} \\
(0.236)\end{array}$ & $\begin{array}{c}-5.963^{* * *} \\
(1.054)\end{array}$ & $\begin{array}{c}-0.248^{* * *} \\
(0.213)\end{array}$ & $\begin{array}{c}-0.499^{* *} \\
(0.221)\end{array}$ \\
\hline Pseudo R-squared & 0.146 & 0.139 & 0.089 & 0.115 & 0.127 & 0.086 & 0.099 & 0.094 \\
\hline Observations & 288 & 2,016 & 61,344 & 252 & 1,764 & 53,676 & 252 & 240 \\
\hline $\begin{array}{l}\text { No. geographical units } \\
\text { Location fixed effects }\end{array}$ & 1 & $\begin{array}{l}7 \\
\sqrt{ }\end{array}$ & $\underset{\sqrt{ }}{213}$ & 1 & $\begin{array}{l}7 \\
\sqrt{ }\end{array}$ & $\underset{V}{213}$ & 1 & 1 \\
\hline
\end{tabular}

The authors declare no competing interests. 